Article

\title{
On the Existence of Solutions of a Two-Layer Green Roof Mathematical Model
}

\author{
J. Ignacio Tello ${ }^{1}$, Lourdes Tello ${ }^{2, *}$ and María Luisa Vilar ${ }^{2}$ \\ 1 Department of Fundamental Mathematics, School of Sciences, UNED, 28040 Madrid, Spain; \\ jtello@mat.uned.es \\ 2 Department of Applied Mathematics, ETS Arquitectura, Universidad Politécnica de Madrid, \\ Av. Juan de Herrera 4, 28040 Madrid, Spain; marialuisa.vilar.guerrero@alumnos.upm.es \\ * Correspondence: 1.tello@upm.es
}

Received: 19 August 2020; Accepted: 15 September 2020; Published: 18 September 2020

check for updates

\begin{abstract}
The aim of this article is to fill part of the existing gap between the mathematical modeling of a green roof and its computational treatment, focusing on the mathematical analysis. We first introduce a two-dimensional mathematical model of the thermal behavior of an extensive green roof based on previous models and secondly we analyze such a system of partial differential equations. The model is based on an energy balance for buildings with vegetation cover and it is presented for general shapes of roofs. The model considers a vegetable layer and the substratum and the energy exchange between them. The unknowns of the problem are the temperature of each layer described by a coupled system of two partial differential equations of parabolic type. The equation modeling the evolution of the temperature of the substratum also considers the change of phase of water described by a maximal monotone graph. The main result of the article is the proof of the existence of solutions of the system which is given in detail by using a regularization of the maximal monotone graph. Appropriate estimates are obtained to pass to the limit in a weak formulation of the problem. The result goes one step further from modeling to validate future numerical results.
\end{abstract}

Keywords: nonlinear mathematical models; green roof models; partial differential equations on manifolds; energy balance models; maximal monotone graphs

\section{Introduction}

A green roof is a roof that contains a soil (growing media) and vegetation layer as its outermost surface. Green roofs are used in buildings since ancient times, they have multiple benefits, among them, the reduction of the effect of the urban heat island, the building energy savings, the storm water reduction, aesthetic effect and the acoustic benefits (see [1-3] and references therein for more details).

Two main types of green roof exist: extensive green roof and intensive green roof. In the extensive green roofs, the depth of the growing media is less than the depth in the intensive green roofs. The choice of the vegetation is also different. Sedum, small grasses, herbs and flowering herbaceous plants which do not need permanent irrigation system are the typical vegetation in an extensive green roof.

During the last few decades, green roofs have been studied from different points of view. Several mathematical models of heat transfer or heat and mass transfer for green roofs have been proposed (see [4] for a review of this type of models). Two of the most representative green roof mathematical models were proposed by del Barrio [5] and Sailor [6]. These models are based on an energy balance. In [5], a set of relevant parameters in the design of green roofs were described, among them, the leaf area index (LAI) or soil density and thickness. The energy balance mathematical model considered in [6] was validated with experimental data of a green roof installed in Florida. 
Moreover, several energy balance models describing the thermal behaviour of green roofs have appeared (e.g., [2]). Most of these energy balance models are one-dimensional models, that is, they consider the substratum and the canopy as homogeneous. See [4] for a review of green roof mathematical models.

In [3] experimental results about the influence of the choice of the vegetation species, the weather conditions and the watering on the cooling potential in prefabricated green roofs were obtained. Experimental data were used in some other works to validate green roof mathematical models (see e.g., $[1,6])$.

Most studies agree that evapotranspiration is one of the main factors that affect the thermal behaviour of green roofs (see [4,7] and the references therein). Evapotranspiration is a combination of soil evaporation and plant transpiration. Comparing the energy balance of a green roof to the energy balance of a conventional roof, we notice that evapotranspiration is not in the second one. Evapotranspiration effect plays an important role in Biosphere models (see [8]).

The purpose of our work is to present a mathematical model of the energy balance of a green roof which includes the shape of the green roof and its mathematical treatment. A system of two coupled nonlinear parabolic equations describing the temperature in a vegetation layer and the temperature in the substrate has been considered. The model includes the main feedback mechanisms of the energy balance than previous models consider, like evapotranspiration. The proposed model in this work considers that the properties of the substratum depend on the temperature, in fact, the equation modeling the evolution of the temperature of the substratum also consider the change of phase of water described by a maximal monotone graph, denoted by $\gamma$. In consequence, the heat capacity of the substratum is not constant, it is a function depending on the temperature. This is the main difficulty in the mathematical treatment of the problem. In the proof of the existence of solutions we have used a regularization of the maximal monotone graph to avoid the lack of regularity produced by $\gamma$. The space domain is a surface $\mathcal{M}$ of $\mathbb{R}^{3}$, then the obtained model is a two dimensional model which allow us to include the shape of the green roof in the space domain; formulation of partial differential equations on Riemannian manifolds is required. Although most of the green roofs are flat we can also find green roofs with different shapes, spherical, cylindrical, or non-standard shape adapted to the terrain. We mention two examples of buildings whose green roofs are not contained in a plane: California Academy of Sciences by the architect Renzo Piano and la Maison Vague (Wave House) by the architect Patrick Nadeau. The model will allow us to analyze the response of the temperature to different choices of shape of the green roof, choice of the vegetation or the influence of the local climate conditions, for example.

The article is organized as follows. In Section 2, we introduce the mathematical model and the required assumptions and simplifications. In Section 3, we study the system of PDEs from a mathematical point of view. After introducing the notion of weak formulation of the equations we proceed to prove the existence of solutions. Finally, Sections 4 and 5 are devoted to discussion and conclusions respectively.

Notice that due to the shape of $\gamma$, we do not expect a regular solution $T_{s}$ in the classical sense, i.e., $T_{s} \notin C_{t, x}^{1,2}((0, T) \times \mathcal{M})$ because the variation of $T_{s}$ with respect to the heat presents a discontinuity at $T_{s}=273 \mathrm{~K}$ due to the change of phase. Since, the existence of a measurable and continuous temperature is expected, as real experiments show, a new notion of solution has to be introduced. In such direction, it is natural to present the notion of weak solution and weak formulation in appropriate functional spaces. Following the original concept of distributions and generalized functions studied by L. Schwartz and the extensive literature existing from the 40s to our days, we introduce the notion of weak solution in Definition 1, Section 3. The weak formulation is an alternative to validate the model, that can not be interpreted in terms of classical solutions and it should be understood in terms of distributions. The model is based on an energy balance, hence the temperature $T_{S}$ is linked to energy. In particular, $T_{S}$ is related to the latent heat of fusion, which is implicitly defined by $\gamma$ and can only be interpreted in terms of distributions. 
To obtain the existence of solutions, we first regularize the problem by using a regularization of $\gamma$ to obtain uniform bounds of $\int_{\mathcal{M}}\left|\nabla T_{\epsilon s}\right|^{2} d A$ independent of $\epsilon$. Such estimates guarantee the pass to the limit in the weak formulation of the approximated problem to the limit one.

\section{A Green Roof Mathematical Model}

In this section we model the energy balance of a green roof in a given geometry, which is not necessarily flat. The model is based on an energy balance and it includes the interactions between these two layers: vegetation and substratum. The model is based on the proposed ones by del Barrio [5] and Sailor [6]. In our case, the space domain considered is a two-dimensional surface, $\mathcal{M}$.

The model describes the evolution of the temperature in two different layers: the vegetable layer and the substratum layer, denoted by $T_{v}$ and $T_{S}$ respectively (temperatures are given in Kelvin).

The substratum is a porous medium. It is a mixture of solid (minerals and organic material), liquid (water) and gas (air and water steam). See De Vries [9] for thermal properties of soils. We consider the graph $\gamma(u)$ in order to include the change of phase in the model and so, the heat capacity of the substratum depends on the temperature.

In the vegetable layer, the energy balance depends on the following mechanisms

- solar radiation absorbed by the vegetation,

- long wave radiation exchange between vegetation and the exterior,

- radiative exchange between the two layers (vegetation and ground),

- the sensible flux between vegetation and the air surrounding the vegetation,

- evapotranspiration,

- conduction of the heat in every layer.

Following Sailor [6], the energy balance in the vegetable layer is given by the function

$$
F_{1}:=\sigma_{v}\left[R_{a v}+\epsilon_{v} R_{i}-\epsilon_{v} \sigma\left|T_{v}\right|^{3} T_{v}\right]+\frac{\sigma_{v} \epsilon_{v} \epsilon_{S} \sigma}{\epsilon_{l}}\left(\left|T_{S}\right|^{3} T_{S}-\left|T_{v}\right|^{3} T_{v}\right)+H_{v}+L_{v},
$$

where

- $\sigma_{v}$ is the fractional vegetation coverage.

- $R_{a v}$ is the absorbed solar radiation (short wave) by the vegetal layer given by

$$
R_{a v}=Q S(t, x) \beta_{v}(t, x)
$$

where $t$ is the time and $x \in \mathcal{M} . Q$ is the solar constant, $S$ is the isolation function (depending on the orientation of the surface). $\beta_{v}$ is the coalbedo in the vegetal layer, i.e., $1-\beta_{v}$ is the albedo function, that is, the fraction which is reflected.

- $R_{i}$ is the long wave radiation which is absorbed.

- $\sigma$ is the Stefan-Boltzman constant.

- $\epsilon_{v}$ is the emissivity of the vegetal layer.

- $\epsilon_{S}$ is the emissivity of the soil layer.

- $\epsilon_{l}:=\epsilon_{s}+\epsilon_{v}-\epsilon_{s} \epsilon_{v}$.

- LAI is the leaf area index (see Frankenstein et al. [10]) defined as follows

$$
L A I:=A_{0}-A_{1}\left[298-T_{S}\right]^{2}
$$

where $A_{0}$ and $A_{1}$ are positive constants in the following ranges

- $\quad A_{0} \in(2,6)$

- $\quad A_{1} \in[0.0016,0.0088]$. 
If we consider small scales of time, the parameter $L A I$ can be consider as constant.

- $H_{v}$ is the sensible heat flux between the vegetal layer and the air (see Frankenstein et al. [10] and references therein). Following [10] we define $H_{v}$ as follows

$$
H_{v}:=\left(e_{0}+1.1 L A I \rho_{a f} c_{p, a} C_{f} W_{a f}\right)\left(T_{a f}-T_{v}\right)
$$

where

- $\quad e_{0}$ windless exchange coefficient for sensible heat $\left(2.0 \mathrm{~W} / \mathrm{m}^{2}\right)$.

- $\quad \rho_{a f}$ is the air density in the foliage $\left(\mathrm{kg} / \mathrm{m}^{3}\right)$ near the atmosphere-foliage interface. It is a given function and for simplicity we assume homogeneous in space, i.e.,

$$
\rho_{a f}(t, x)=\rho_{a f}(t) .
$$

- $\quad C_{f}$ bulk transfer coefficient;

- $\quad T_{a f}$ is the air temperature in the foliage approximated by

$$
T_{a f}=\left(1-\sigma_{v}\right) T_{a}+\sigma_{v}\left(0.3 T_{a}+0.6 T_{v}+0.3 T_{s}\right)
$$

and $T_{a}$ is the air temperature measured at the shelter (see Frankenstein et al. [10] for more details).

- $\quad W_{a f}$ wind speed at the air/foliage interface $(\mathrm{m} / \mathrm{s})$.

- $\quad c_{p, a}$ specific heat of air at constant pressure.

- $\quad L_{v}$ is the latent heat flux expressed in terms of the unknown temperature. We notice that $L_{v}$ depends on the stomatal resistance coefficient of the leaves of the vegetal layer.

$$
L_{v}=l_{v} L A I \rho_{a f} C_{f} W_{a f} \hat{r}\left(q_{a s}-q_{f, s a t}\right)
$$

where

- $\quad l_{v}$ is the latent heat of vaporization, it is the amount of energy required to convert a unit mass of water to steam. It is measured in units of $\mathrm{J} / \mathrm{kg}$ and is inversely proportional to the temperature. From Henderson-Sellers [11] it is estimated as follows (see also Sailor [6])

$$
l_{v}=1.911846 \times 10^{6} \frac{T_{v}^{2}}{\left(T_{v}-33.91\right)^{2}} .
$$

- $\quad \rho_{a f}, C_{f}, W_{a f}$ have been defined previously.

- $\quad \hat{r}$ is given by

$$
\hat{r}=\frac{L A I}{L A I+r_{0}},
$$

and $r_{0}$ is a given constant (see [10] for more details).

- $\quad q_{a s}$ and $q_{f, s a t}$ are the mixing ratio of the air within the canopy and the saturation mixing ratio at the foliage surface temperature. The explicit expression can be found in [6]. We assume that it is a given function.

For simplicity we assume

$$
L_{v}=A_{0}-A_{1}\left[298-T_{v}\right]^{2} .
$$

The energy balance in the substratum is given by $F_{2}$ (see Sailor [6])

$$
F_{2}:=\left(1-\sigma_{v}\right)\left[R_{a s}+\epsilon_{s} R_{i}-\epsilon_{s}\left|T_{s}\right|^{3} T_{s}\right]-\frac{\sigma_{v} \epsilon_{v} \epsilon_{S} \sigma}{\epsilon_{l}}\left(\left|T_{s}\right|^{3} T_{s}-\left|T_{\mathcal{v}}\right|^{3} T_{v}\right)+H_{s}+L_{s},
$$


where

- $R_{a s}$ is the absorbed solar radiation by the substratum layer,

$$
R_{a s}=Q S(t, x) \beta_{s}(t, x),
$$

and $\beta_{s}$ represents the coalbedo function of the substratum layer.

- $H_{s}$ is the sensible heat flux

$$
H_{s}=\left(e_{0}+\rho_{a g} c_{p, a} C_{h g} W_{a f}\right)\left(T_{a f}-T_{s}\right)
$$

where

- $\quad e_{0}$ have been already defined.

- The bulk transfer coefficient for sensible heat $C_{h}^{g}$ is given as follows (see also [10])

$$
C_{h g}=\left\{\begin{array}{lll}
\frac{A_{6}}{\left(1-16 R_{i b}\right)^{\frac{1}{2}}} & \text { if } & T_{v}<\frac{\left(1-0.3 \sigma_{v}\right) T_{s}+\left(0.7 \sigma_{v}-1\right) T_{a}}{0.6 \sigma_{v}} ; \\
\frac{A_{6}}{1-5 R_{i b}} & \text { if } & T_{v} \geq \frac{\left(1-0.3 \sigma_{v}\right) T_{s}+\left(0.7 \sigma_{v}-1\right) T_{a}}{0.6 \sigma_{v}} ;
\end{array}\right.
$$

and

$$
R_{i b}:=A_{7} \frac{T_{a f}-T_{s}}{T_{a f}+T_{s}}
$$

for $A_{7}=\frac{2 g Z_{a}}{W_{a f}^{2}}$.

In the model, $C_{h g}$ is assumed as a given function.

- $\quad T_{a f}$ air temperature in the foliage approximated by (see Frankenstein et al. [10] and references therein)

$$
T_{a f}=\left(1-\sigma_{v}\right) T_{a}+\sigma_{v}\left(0.3 T_{a}+0.6 T_{v}+0.3 T_{s}\right)
$$

and $T_{a}$ is the air temperature measured at the shelter (known data).

- $\quad W_{a f}$ wind speed at the air/foliage interface $(\mathrm{m} / \mathrm{s})$

- $\quad c_{p, a}$ specific heat of air at constant pressure.

Then

$$
H_{s}=\left(\tilde{B}_{0}+B_{4} C_{h g}\right)\left(\tilde{B}_{1}+\tilde{B}_{2} T_{v}+\tilde{B}_{3} T_{s}\right) .
$$

Notice that $C_{h g}$ is assumed as a given function, therefore $H_{s}$ becomes

$$
H_{s}=B_{1}+B_{2} T_{v}+B_{3} T_{s}
$$

- $\quad L_{S}$ is the latent heat flux. Following Sailor [6] we have

$$
L_{s}=C_{e, s} l_{s} W_{a f} \rho_{a s}\left(q_{a s}-q_{s}\right),
$$

where

- $C_{e, s}$ is the bulk transfer coefficient, it is analogous to $C_{f}$, assumed constant.

- $\quad l_{s}$ is the latent heat of vaporization at the ground surface temperature, assumed constant.

- $\quad W_{a, f}$ has been previously defined as the wind speed at the air/foliage interface and it is a given datum.

- $\quad \rho_{a, s}$ is the air density near the soil surface. It is a known datum. 
- $\quad q_{a s}$ and $q_{s}$ are the mixing ratio at the foliage-atmosphere interface and the mixing ratio at the ground surface. We assume they are known data. The explicit expression is presented in $[6,10]$.

Then, $L_{s}$ is assumed a known function, i.e.,

$$
L_{S}=L_{S}(t, x)
$$

Notice that $F_{1}$ and $F_{2}$ depend on the type of vegetation, in particular, they depend on two functions which characterizes the plants used in the green roof: Leaf Area Index (LAI) and Fractional vegetation coverage $\left(\sigma_{v}\right)$. If we consider small scales of time, these functions (LAI and $\sigma_{v}$ ) can be included in the model as constant. We notice that they are two of the relevant parameters of the model.

One of the characteristics of the energy balance of the green roof is the solar shading produced by the foliage and the cooling by evapotranspiration (these two effects are not in the energy balance of the conventional roof). We consider some relevant parameters related with vegetation: leaf area index (LAI), foliage fractional coverage $\left(\sigma_{v}\right)$, vegetation stomatal resistance $\left(r_{s}\right)$ and the vegetation coalbedo $\beta_{v}$. These parameters could depend on the vegetation type and the seasons. The analysis of the sensitivity of the model front fluctuations of these parameters would be a useful future research.

The model also considers the heat conduction in every layer, whose coefficients are given by $k_{1}$, $k_{2}$ respectively. Considering the heat conduction and the energy balance, under suitable boundary and initial conditions, we arrive to following system of partial differential equations

$$
\begin{cases}C_{v} \frac{\partial T_{v}}{\partial t}-\operatorname{div}\left(k_{1}(x) \nabla_{\mathcal{M}} T_{v}\right)=F_{1}\left(T_{v}, T_{s}\right), & \text { in } \mathcal{M}_{T} \\ \frac{\partial \gamma\left(T_{s}\right)}{\partial t}-\operatorname{div}\left(k_{2}(x) \nabla_{\mathcal{M}} T_{s}\right) \ni F_{2}\left(T_{v}, T_{s}\right), & \text { in } \mathcal{M}_{T} \\ T_{v}=g_{v}, & \text { in } \partial \mathcal{M}_{T} \\ T_{s}=g_{s}, & \text { in } \partial \mathcal{M}_{T} \\ T_{v}(0, x)=T_{v 0}(x), & \text { in } \mathcal{M} \\ T_{S}(0, x)=T_{s 0}(x), & \text { in } \mathcal{M}\end{cases}
$$

where

$$
\mathcal{M}_{T}:=\{(t, x) \in(0, T) \times \mathcal{M}\}
$$

and

$$
\partial \mathcal{M}_{T}:=\{(t, x) \in(0, T) \times \partial \mathcal{M}\} .
$$

Here, the heat capacity in every layer could depend on the moisture.

$\gamma$ is defined as the graph

$$
\gamma(s)= \begin{cases}\gamma_{1}(s-273), & s<273 \\ {[0, L],} & s=273 \\ \gamma_{2}(s-273)+L & s>273\end{cases}
$$

for $\gamma_{1}$ and $\gamma_{2}$ positive numbers. These constants could depend on the moisture. See the climate model studied in [12] for more details. See also [13].

Notice that $F_{1}$ and $F_{2}$ can be expressed as follows

$$
\begin{gathered}
F_{1}\left(T_{v}, T_{S}\right)=a_{0}\left(\left|T_{S}\right|^{3} T_{S}-\left|T_{v}\right|^{3} T_{v}\right)-a_{1}\left|T_{\mathcal{v}}\right|^{3} T_{\mathcal{v}} \\
+\left[a_{2}-\left(268-T_{S}\right)^{2}\right]\left(a_{3}-a_{4} T_{v}+a_{5} T_{S}\right)+a_{6}+a_{7}\left[298-T_{S}\right]^{2}
\end{gathered}
$$

and 


$$
F_{2}\left(T_{v}, T_{s}\right)=a_{0}\left(\left|T_{v}\right|^{3} T_{v}-\left|T_{s}\right|^{3} T_{s}\right)-b_{1}\left|T_{s}\right|^{3} T_{s}+b_{2} T_{v}+b_{3} T_{s}+b_{4}
$$

\section{Well-Posed Problem}

We start the mathematical treatment of the obtained model by analyzing the existence of solutions under the following assumptions:

$\left(\mathcal{H}_{M}\right) \quad \mathcal{M}$ is a two-dimensional connected oriented Riemannian $C^{\infty}$ manifold with compact closure and regular boundary $\partial \mathcal{M}$.

$\left(\mathcal{H}_{S}\right) \quad S:[0,+\infty) \times \mathcal{M} \rightarrow \mathbb{R}, S \in L^{\infty}((0,+\infty) \times \mathcal{M})$ such that there exist a positive constant $s_{1}$ verifying $0 \leq S(t, x) \leq s_{1}$.

$\left(\mathcal{H}_{\beta}\right) \quad \beta_{v} \in L^{\infty}((0,+\infty) \times \mathcal{M}), \beta_{s} \in L^{\infty}((0,+\infty) \times \mathcal{M}), 0 \leq \beta_{v}(t, x) \leq 1,0 \leq \beta_{s}(t, x) \leq 1$.

$\left(\mathcal{H}_{v}\right) \quad H_{v}=H_{v}\left(t, x, T_{v}, T_{s}\right), H_{v} \in C([0,+\infty) \times \overline{\mathcal{M}} \times \mathbb{R} \times \mathbb{R})$.

$\left(\mathcal{H}_{s}\right) \quad H_{s}=H_{s}\left(t, x, T_{v}, T_{s}\right), H_{s} \in C([0,+\infty) \times \overline{\mathcal{M}} \times \mathbb{R} \times \mathbb{R})$.

$\left(\mathcal{H}_{L}\right) \quad L_{v}=L_{v}\left(t, x, T_{v}, T_{s}\right), L_{s}=L_{s}\left(t, x, T_{v}, T_{s}\right), L_{v}, L_{s} \in C([0,+\infty) \times \overline{\mathcal{M}} \times \mathbb{R} \times \mathbb{R})$.

$\left(H_{\gamma}\right) \quad \gamma$ is a maximal monotone graph defined by $\gamma(s)=\gamma_{1}(s-273)$ if $s<273, \gamma(s)=\gamma_{2}(s-273)+L$ if $s>273$ and $\gamma(s)=[0, L]$ if $s=273$, where $\gamma_{1}, \gamma_{2}$ and $L$ are positive constants.

$\left(H_{F}\right) \quad F_{1}$ and $F_{2}$ are defined in (7) and (8), resp. whose coefficients $a_{i}=a_{i}(t, x)(i=2, . ., 7)$ and $b_{j}=b_{j}(t, x)(j=2, . ., 4)$ are uniformly bounded and $a_{0}, a_{1}$ and $b_{1}$ are positive constants.

$\left(\mathcal{H}_{k}\right)$ The heat conduction coefficients $k_{1}$ and $k_{2}$ satisfy

$$
k_{i} \in W^{1, \infty}(\mathcal{M}), \quad i=1,2
$$

and there exists a constants $k_{0}>0$ such that

$$
0<k_{0} \leq k_{i} \leq k_{0}^{-1}, \quad i=1,2 .
$$

$\left(\mathcal{H}_{g}\right) \quad$ The boundary conditions $g_{v}$ and $g_{s}$ satisfy

$$
g_{v}, g_{s} \in C^{2}([0, T] \times \partial \mathcal{M})
$$

and

$$
g_{v} \geq 0, \quad g_{s} \geq 0 .
$$

$\left(\mathcal{H}_{p}\right)$ The constants $\sigma_{v}, \epsilon_{v}, \epsilon_{s}, \sigma, \lambda, C_{v}, Q$ are positive.

$\left(\mathcal{H}_{0}\right)$ The initial data $T_{0 v}, T_{0 s} \in H^{1}(\mathcal{M}) \cap L^{\infty}(\mathcal{M})$, and satisfy the boundary condition

$$
T_{0 v}=g_{v}(0, x), \quad T_{0 s}=g_{s}(0, x), \quad \text { in } x \in \partial \mathcal{M} .
$$

Notice that divergence and gradient in the diffusion terms are understood in the sense of the Riemannian metric of $\mathcal{M}$. In particular if $k_{1} \equiv 1$ then the diffusion operator $\operatorname{div}\left(\nabla_{\mathcal{M}} T_{v}\right)$ is the Laplace-Beltrami operator of $T_{v}$ (see [14]). For simplicity in the notation we denote $\nabla_{\mathcal{M}}$ by $\nabla$. See also [15] where an energy balance model on a Riemannian manifold was considered. For maximal monotone operators properties see [16]. Now, following [14] we define the functional spaces on manifolds,

$$
L^{p}(\mathcal{M}):=\left\{u: \mathcal{M} \rightarrow \mathbb{R} \text { measurable, } \int_{\mathcal{M}}|u|^{p} d A<+\infty\right\},
$$

for $1 \leq p<\infty$

$$
L^{\infty}(\mathcal{M}):=\left\{u: \mathcal{M} \rightarrow \mathbb{R} \text { measurable, ess } \sup _{\mathcal{M}}|u|<+\infty\right\}
$$


Denote by $T_{\mathbf{p}} \mathcal{M}$ the tangent space in every point $\mathbf{p} \in \mathcal{M}$ and define the bundle tangent space $T \mathcal{M}=\cup_{p \in \mathcal{M}} T_{\mathbf{p}} \mathcal{M}$. The space $L^{p}(T \mathcal{M})$, also denoted by $L^{p}(\mathcal{M}, T \mathcal{M})$, is defined by

$$
L^{p}(T \mathcal{M}):=\left\{X: \mathcal{M} \rightarrow T \mathcal{M}: \int_{\mathcal{M}}|<X, X>|^{\frac{p}{2}} d A<+\infty\right\}
$$

where $<\cdot, \cdot>$ is the scalar product in $T \mathcal{M}$ given by the Riemannian metrics on $\mathcal{M}$.

$$
W^{1, p}(\mathcal{M}):=\left\{u \in L^{p}(\mathcal{M}): \nabla u \in L^{p}(T \mathcal{M})\right\} .
$$

In particular, $H^{1}(\mathcal{M})=W^{1,2}(\mathcal{M})$.

$$
W^{1, \infty}(\mathcal{M}):=\left\{u: \mathcal{M} \rightarrow \mathbb{R} \text { measurable, ess } \sup _{\mathcal{M}}|u|+\text { ess } \sup _{\mathcal{M}}|\nabla u|<+\infty\right\} .
$$

We also define $L^{2}\left(0, T: H^{1}(\mathcal{M})\right)$ as the set of measurable functions $u:(0, T) \times \mathcal{M} \rightarrow \mathbb{R}$ verifying

$$
\int_{0}^{T} \int_{\mathcal{M}}|u|^{2} d A d t+\int_{0}^{T} \int_{\mathcal{M}}|\nabla u|^{2} d A d t<+\infty .
$$

See $[14,15,17]$ for more details about functional spaces defined on manifolds.

We now introduce the notion of weak solution.

Definition 1. We say that $\left(T_{v}, T_{S}\right) \in\left[L^{2}\left(0, T: H^{1}(\mathcal{M})\right)\right]^{2}$ is a weak solution of (5) if

(i) $\left(T_{v}, T_{s}\right) \in\left[L^{\infty}\left(\mathcal{M}_{T}\right)\right]^{2}$.

(ii) $\left(T_{v}, T_{s}\right)=\left(g_{v}, g_{s}\right)$ for $x \in \partial \mathcal{M}$.

(iii) There exists $Z \in L^{\infty}\left(\mathcal{M}_{T}\right), Z \in \gamma\left(T_{s}\right)$ such that

$$
\begin{gathered}
\int_{\mathcal{M}} C_{v} T_{v}(T, x) \psi_{1}(T, x) d A-\int_{\mathcal{M}} C_{v} T_{v}(0, x) \psi_{1}(0, x) d A-\int_{0}^{T} \int_{\mathcal{M}} C_{v} T_{v} \psi_{1} d A d t \\
+\int_{0}^{T} \int_{\mathcal{M}} k_{1}(x) \nabla T_{v} \nabla \psi_{1} d A d t=\int_{0}^{T} \int_{\mathcal{M}} F_{1}\left(T_{S}, T_{v}\right) \psi_{1} d A d t
\end{gathered}
$$

and

$$
\begin{gathered}
\int_{\mathcal{M}} Z(T, x) \psi_{2}(T, x) d A-\int_{\mathcal{M}} Z(0, x) \psi_{2}(0, x) d A-\int_{0}^{T} \int_{\mathcal{M}} Z \psi_{2 t} d A d t \\
+\int_{0}^{T} \int_{\mathcal{M}} k_{2}(x) \nabla T_{\mathcal{V}} \nabla \psi_{2} d A d t=\int_{0}^{T} \int_{\mathcal{M}} F_{2}\left(T_{s}, T_{\mathcal{V}}\right) \psi_{2} d A d t
\end{gathered}
$$

for $\left(\psi_{1}, \psi_{2}\right) \in\left[L^{2}\left(0, T: H_{0}^{1}(\mathcal{M})\right) \cap H^{1}\left(0, T: L^{2}(\mathcal{M})\right)\right]^{2}$.

We remark that although $\gamma$ is multivalued, in order to solve the problem we introduce the following approximation of $\gamma$, denoted by $\gamma_{\epsilon}$ for $\epsilon>0$ in the following way

$$
\gamma_{\epsilon}(s)=\left\{\begin{array}{l}
\gamma_{1}(s-273), \text { if } s<273 \\
-\left(\left(\gamma_{2}-\gamma_{1}\right) \epsilon+2 L\right) \frac{(s-273)^{3}}{\epsilon^{3}}+\left(3 L+2 \epsilon\left(\gamma_{2}-\gamma_{1}\right)\right) \frac{(s-273)^{2}}{\epsilon^{2}}+\gamma_{1}(s-273), \\
\text { if } 273 \leq s \leq 273+\epsilon ; \\
\gamma_{2}(s-273)+L, \text { if } s>273+\epsilon .
\end{array}\right.
$$

Notice that $\gamma_{\epsilon} \in C^{1}$ and there exists a positive constant $c(\epsilon)$ such that $\left|\gamma_{\epsilon}^{\prime \prime}\right|<c(\epsilon)$ a.e.

We denote the inverse of $\gamma_{\epsilon}$ by $\varphi_{\epsilon}$, i.e., $\varphi_{\epsilon}$ satisfies

$$
\varphi_{\epsilon}\left(\gamma_{\epsilon}(s)\right)=s
$$


Consider the approximated problem

$$
\begin{cases}C_{v} \frac{\partial T_{\epsilon v}}{\partial t}-\operatorname{div}\left(k_{1}(x) \nabla_{\mathcal{M}} T_{\epsilon v}\right)=F_{1}\left(T_{\epsilon v}, \varphi_{\epsilon}\left(\Gamma_{\epsilon}\right)\right), & \text { in } \mathcal{M}_{T}, \\ \frac{\partial \Gamma_{\epsilon}}{\partial t}-\operatorname{div}\left(k_{2}(x) \nabla_{\mathcal{M}} \varphi_{\epsilon}\left(\Gamma_{\epsilon}\right)\right)=F_{2}\left(T_{\epsilon v}, \varphi_{\epsilon}\left(\Gamma_{\epsilon}\right)\right), & \text { in } \mathcal{M}_{T} \\ T_{\epsilon v}=g_{v}, & \text { in } \partial \mathcal{M}_{T} \\ \Gamma_{\epsilon}=\gamma_{\epsilon}\left(g_{s}\right), & \text { in } \partial \mathcal{M}_{T} \\ T_{\epsilon v}(0, x)=T_{v 0}(x), & \text { in } \mathcal{M}, \\ \Gamma_{\epsilon}(0, x)=\gamma_{\epsilon}\left(T_{s 0}\right), & \text { in } \mathcal{M} .\end{cases}
$$

We now introduce the notion of weak solution of problem (10)

Definition 2. We say that $\left(T_{\epsilon v}, \Gamma_{\epsilon}\right) \in\left[C\left(0, T: L^{2}(\mathcal{M})\right)\right]^{2}$ is a weak solution of (10) if

(i) $\left(T_{\epsilon v}, \Gamma_{\epsilon}\right) \in\left[L^{\infty}\left(\mathcal{M}_{T}\right) \cap L^{2}\left(0, T: H^{1}(\mathcal{M})\right)\right]^{2}$,

(ii) $\left(T_{\epsilon v}, \Gamma_{\epsilon}\right)=\left(g_{v}, \gamma_{\epsilon}\left(g_{s}\right)\right)$ for $x \in \partial \mathcal{M}$, such that

$$
\begin{gathered}
\int_{\mathcal{M}} T_{\epsilon v}(T, x) \psi_{1}(T, x) d A-\int_{\mathcal{M}} T_{\epsilon v}(0, x) \psi_{1}(0, x) d A-\int_{0}^{T} \int_{\mathcal{M}} T_{\epsilon v} \psi_{1 t} d A d t \\
+\int_{0}^{T} \int_{\mathcal{M}} k_{1}(x) \nabla T_{\epsilon v} \nabla \psi_{1} d A d t=\int_{0}^{T} \int_{\mathcal{M}} F_{1}\left(T_{\epsilon v}, \varphi_{\epsilon}\left(\Gamma_{\epsilon}\right)\right) \psi_{1} d A d t
\end{gathered}
$$

and

$$
\begin{aligned}
& \int_{\mathcal{M}} \Gamma_{\epsilon}(T, x) \psi_{2}(T, x) d A-\int_{\mathcal{M}} \Gamma_{\epsilon}(0, x) \psi_{2}(0, x) d A-\int_{0}^{T} \int_{\mathcal{M}} \Gamma_{\epsilon} \psi_{2 t} d A d t \\
& +\int_{0}^{T} \int_{\mathcal{M}} k_{2}(x) \nabla \varphi_{\epsilon}\left(\Gamma_{\epsilon}\right) \nabla \psi_{2} d A d t=\int_{0}^{T} \int_{\mathcal{M}} F_{2}\left(T_{\epsilon v}, \varphi_{\epsilon}\left(\Gamma_{\epsilon}\right)\right) \psi_{2} d A d t
\end{aligned}
$$

for $\left(\psi_{1}, \psi_{2}\right) \in\left[L^{2}\left(0, T: H_{0}^{1}(\mathcal{M})\right) \cap H^{1}\left(0, T: L^{2}(\mathcal{M})\right)\right]^{2}$.

Theorem 1. Assume the hypotheses $\left(\mathcal{H}_{M}\right)-\left(\mathcal{H}_{p}\right)$ then, for every initial datum $\left(T_{v}^{0}, T_{s}^{0}\right)$ satisfying $\left(\mathcal{H}_{0}\right)$ the problem (5) has a weak solution

$$
\left(T_{v}, T_{s}\right) \in\left[C\left(0, T: L^{2}(\mathcal{M})\right) \cap L^{\infty}\left(\mathcal{M}_{T}\right) \cap L^{2}\left(0, T: H^{1}(\mathcal{M})\right)\right]^{2} .
$$

The proof of Theorem 1 is organized into several steps.

We consider the function $w=\left(w_{1}, w_{2}\right)$ defined as follows

$$
\begin{cases}-\operatorname{div}\left(k_{1}(x) \nabla w_{1}\right)=0, & x \in \mathcal{M}, \\ w_{1}=g_{v}, & x \in \partial \mathcal{M},\end{cases}
$$

and

$$
\begin{cases}-\operatorname{div}\left(k_{2}(x) \nabla w_{2}\right)=0, & x \in \mathcal{M}, \\ w_{2}=g_{s}, & x \in \partial \mathcal{M} .\end{cases}
$$

Notice that under assumptions $\left(\mathcal{H}_{k}\right)-\left(\mathcal{H}_{g}\right)$ we have that

$$
w \in\left[L^{2}\left(0, T: W^{2, p}(\mathcal{M})\right) \cap H^{1}\left(0, T: W^{1, p}(\mathcal{M})\right)\right]^{2}, \quad \text { for any } p<\infty,
$$

see Gilbart and Trudinger [18], Theorem 9.15 p. 241.

We first obtain the following a-priori estimates.

Lemma 1. Let $\left(T_{\epsilon v}, \Gamma_{\epsilon}\right)$ the solution to (10), and $T_{\epsilon s}=\varphi_{\epsilon}\left(\Gamma_{\epsilon}\right)$ then, under assumptions of Theorem 1 , then, for any $T<\infty$ we have that

$$
\left(T_{\epsilon v}, T_{\epsilon s}\right) \in\left[L^{\infty}\left(\mathcal{M}_{T}\right)\right]^{2},
$$


moreover there exists a positive constant $c_{\infty}$ independent of $\epsilon$ and $T$ such that

$$
\left\|T_{\epsilon v}\right\|_{L^{\infty}(\mathcal{M})},\left\|T_{\epsilon s}\right\|_{L^{\infty}(\mathcal{M})} \leq c_{\infty} .
$$

Proof. We denote by $P_{v}$ and $P_{S}$ the following functions

$$
P_{v}\left(T_{\epsilon v}, T_{\epsilon S}\right)=-a_{1}\left|T_{\epsilon v}\right|^{3} T_{\epsilon v}+\left[a_{2}-\left(268-T_{\epsilon S}\right)^{2}\right]\left(a_{3}-a_{4} T_{\epsilon v}+a_{5} T_{\epsilon S}\right)+a_{6}+a_{7}\left[298-T_{\epsilon s}\right]^{2}
$$

and

$$
P_{S}\left(T_{\epsilon v}, T_{\epsilon S}\right)=-b_{1}\left|T_{\epsilon S}\right|^{3} T_{\epsilon S}+b_{2} T_{\epsilon v}+b_{3} T_{\epsilon S}+b_{4}
$$

Let

$$
k:=\max \left\{\sup _{(t, x) \in \mathcal{M}_{T}}\left\{\left|w_{1}\right|\right\}, \sup _{(t, x) \in \mathcal{M}_{T}}\left\{\left|w_{2}\right|\right\}, k_{0}\right\} .
$$

where $k_{0}>0$ satisfies, for $|s| \leq k_{0}+1$

$$
P_{v}\left(T_{\epsilon v}, s\right)<0 \text {, for any } T_{\epsilon v}>k_{0}, \quad P_{v}\left(T_{\epsilon v}, s\right)>0 \text { for any } T_{\epsilon v}<-k_{0},
$$

and

$$
P_{S}\left(s, T_{\epsilon S}\right)<0 \text {, for any } T_{\epsilon S}>k_{0}, \quad P_{v}\left(s, T_{\epsilon s}\right)>0 \quad \text { for any } T_{\epsilon S}<-k_{0} \text {. }
$$

Since

$$
\left|T_{\epsilon s}\right| \leq\left(T_{\epsilon s}-k\right)_{+}+\left(-k-T_{\epsilon s}\right)_{+}+k
$$

we have

$$
F_{1}\left(T_{\epsilon v}, T_{\epsilon S}\right)\left(T_{\epsilon v}-k\right)_{+} \leq \alpha_{1}\left(T_{\epsilon v}, T_{\epsilon S}\right)\left[\left(T_{\epsilon v}-k\right)_{+}^{2}+\left(-T_{\epsilon v}-k\right)_{+}^{2}+\left(T_{\epsilon S}-k\right)_{+}^{2}+\left(-T_{\epsilon S}-k\right)_{+}^{2}\right] .
$$

In similar way we obtain

$$
\begin{gathered}
F_{2}\left(T_{\epsilon v}, T_{\epsilon s}\right)\left(T_{\epsilon s}-k\right)_{+} \leq \alpha_{2}\left(T_{\epsilon v}, T_{\epsilon s}\right)\left[\left(T_{\epsilon S}-k\right)_{+}^{2}+\left(-T_{\epsilon s}-k\right)_{+}^{2}+\left(T_{\epsilon v}-k\right)_{+}^{2}+\left(-T_{\epsilon v}-k\right)_{+}^{2}\right], \\
-F_{1}\left(T_{\epsilon v}, T_{\epsilon s}\right)\left(-T_{\epsilon v}-k\right)_{+} \leq \alpha_{3}\left(T_{\epsilon s}, T_{\epsilon v}\right)\left[\left(T_{\epsilon s}-k\right)_{+}^{2}+\left(-T_{\epsilon s}-k\right)_{+}^{2}+\left(T_{\epsilon v}-k\right)_{+}^{2}+\left(-T_{\epsilon v}-k\right)_{+}^{2}\right]
\end{gathered}
$$

and

$$
-F_{2}\left(T_{\epsilon v}, T_{\epsilon S}\right)\left(-T_{\epsilon S}-k\right)_{+} \leq \alpha_{4}\left(T_{\epsilon v}, T_{\epsilon S}\right)\left[\left(T_{\epsilon v}-k\right)_{+}^{2}+\left(-T_{\epsilon v}-k\right)_{+}^{2}+\left(T_{\epsilon S}-k\right)_{+}^{2}+\left(-T_{\epsilon S}-k\right)_{+}^{2}\right] .
$$

We introduce the truncation function $\tau$ defined by

$$
\tau(s)= \begin{cases}-k, & \text { if } s<-k-1, \\ s, & \text { if }-k-1 \leq s \leq k+1, \\ k+1, & \text { if } k+1<s\end{cases}
$$

and consider the problem (10) where $F_{1}$ and $F_{2}$ are replaced by $\tilde{F}_{1}$ and $\tilde{F}_{2}$

$$
\begin{gathered}
\tilde{F}_{1}=a_{0}\left(\left|\tau\left(T_{\epsilon S}\right)\right|^{3} T_{\epsilon S}-\left|\tau\left(T_{\epsilon v}\right)\right|^{3} T_{\epsilon v}\right)-a_{1}\left|\tau\left(T_{\epsilon v}\right)\right|^{3} T_{\epsilon v} \\
+\left[a_{2}-\left(268-\tau\left(T_{\epsilon s}\right)\right)^{2}\right]\left(a_{3}-a_{4} T_{\epsilon v}+a_{5} T_{\epsilon s}\right)+a_{6}+a_{7}\left[298-\tau\left(T_{\epsilon s}\right)\right]^{2} .
\end{gathered}
$$

and

$$
\tilde{F}_{2}\left(T_{\epsilon v}, T_{\epsilon S}\right)=a_{0}\left(\left|\tau\left(T_{\epsilon v}\right)\right|^{3} T_{\epsilon v}-\left|\tau\left(T_{\epsilon S}\right)\right|^{3} T_{\epsilon S}\right)-b_{1}\left|\tau\left(T_{\epsilon S}\right)\right|^{3} T_{\epsilon S}+b_{2} T_{\epsilon v}+b_{3} T_{\epsilon S}+b_{4} .
$$


Notice that, thanks to Mean Value Theorem,

$$
a_{0}\left(\left|\tau\left(T_{\epsilon S}\right)\right|^{3} T_{\epsilon S}-\left|\tau\left(T_{\epsilon v}\right)\right|^{3} T_{\epsilon v}\right)\left(T_{\epsilon v}-k\right)_{+} \leq 4 a_{0}(k+1)^{3}\left(T_{\epsilon v}-k\right)_{+}\left(T_{\epsilon S}-k\right)_{+}
$$

and

$$
a_{0}\left(\left|\tau\left(T_{\epsilon v}\right)\right|^{3} T_{\epsilon v}-\left|\tau\left(T_{\epsilon S}\right)\right|^{3} T_{\epsilon S}\right)\left(T_{\epsilon S}-k\right)_{+} \leq 4 a_{0}(k+1)^{3}\left(T_{\epsilon S}-k\right)_{+}\left(T_{\epsilon v}-k\right)_{+}
$$

and their symmetric once we multiply by $-\left(-T_{\epsilon v}-k\right)_{+}$and $-\left(-T_{\epsilon v}-k\right)_{+}$. We multiply by $\left(T_{\epsilon v}-k\right)_{+}$ and $-\left(-T_{\epsilon v}-k\right)_{+}$in the first equation of (10) and by $\left(T_{\epsilon S}-k\right)_{+}$and $-\left(-T_{\epsilon S}-k\right)_{+}$in the second equation to obtain, after integration by parts

$$
\begin{gathered}
\frac{d}{d t} \int_{\mathcal{M}}\left[\left(T_{\epsilon v}-k\right)_{+}^{2}+\left(-T_{\epsilon v}-k\right)_{+}^{2}+\left(T_{\epsilon S}-k\right)_{+}^{2}+\left(-T_{\epsilon S}-k\right)_{+}^{2}\right] d A \\
\leq \int_{\mathcal{M}} \alpha\left(\tau\left(T_{\epsilon v}\right), \tau\left(T_{\epsilon S}\right)\right)\left[\left(T_{\epsilon v}-k\right)_{+}^{2}+\left(-T_{\epsilon v}-k\right)_{+}^{2}+\left(T_{\epsilon S}-k\right)_{+}^{2}+\left(-T_{\epsilon S}-k\right)_{+}^{2}\right] d A .
\end{gathered}
$$

Then, Gronwall's Lemma and the choice of the truncation function end the proof.

Lemma 2. Let $\left(T_{\epsilon v}, \Gamma_{\epsilon}\right)$ the solution to (10), and $T_{\epsilon s}=\varphi_{\epsilon}\left(\Gamma_{\epsilon}\right)$ then, under assumptions of Theorem 1, there exists $c>0$ independent of $\epsilon$ and $T$ such that

$$
\int_{0}^{T} \int_{\mathcal{M}}\left(\left|\nabla T_{\epsilon v}\right|^{2}+\left|T_{\epsilon v}\right|^{2}\right) d A d t+\int_{0}^{T} \int_{\mathcal{M}}\left(\left|\nabla T_{\epsilon s}\right|^{2}+\left|T_{\epsilon s}\right|^{2}\right) d A d t \leq c(T+1) .
$$

Proof. We multiply the first equation in (10) by $T_{\epsilon v}-w_{1}$ and integrate by parts over $\mathcal{M}$ to get

$$
\begin{gathered}
\frac{C_{v}}{2} \frac{d}{d t} \int_{\mathcal{M}}\left(T_{\epsilon v}-w_{1}\right)^{2} d A+\int_{\mathcal{M}} k_{1}(x)\left|\nabla\left(T_{\epsilon v}-w_{1}\right)\right|^{2} d A \leq \int_{\mathcal{M}} F_{1}\left(T_{\epsilon v}, T_{\epsilon s}\right)\left(T_{\epsilon v}-w_{1}\right) d A \\
-C_{v} \int_{\mathcal{M}} w_{1 t}\left(T_{\epsilon v}-w_{1}\right) d A .
\end{gathered}
$$

In view of Lemma 1 we have that

$$
\left|F_{1}\left(T_{\epsilon v}, T_{\epsilon S}\right) T_{\epsilon v}\right| \leq c
$$

After integration we obtain

$$
\frac{C_{v}}{2} \int_{\mathcal{M}}\left(T_{\epsilon v}-w_{1}\right)^{2} d A+\int_{\mathcal{M}_{T}} k_{1}(x)\left|\nabla\left(T_{\epsilon v}-w_{1}\right)\right|^{2} d A \leq c(T+1) .
$$

In the same way, we multiply by $T_{\epsilon s}-w_{2}$ in the second equation in (10). Then, after integration over $\mathcal{M}$ we have

$$
\int_{\mathcal{M}} \gamma_{\epsilon}^{\prime}\left(T_{\epsilon s}\right)\left(T_{\epsilon s}\right)_{t}\left(T_{\epsilon s}-w_{2}\right) d A+\int_{\mathcal{M}} k_{2}(x)\left|\nabla\left(T_{\epsilon s}-w_{2}\right)\right|^{2} d A \leq \int_{\mathcal{M}} F_{2}\left(T_{\epsilon v}, T_{\epsilon s}\right)\left(T_{\epsilon s}-w_{2}\right) d A .
$$

The subscript $t$ represents the time derivative. We can express the first term by using

$$
\gamma_{\epsilon}^{\prime}\left(T_{\epsilon S}\right)\left(T_{\epsilon S}\right)_{t}\left(T_{\epsilon S}-w_{2}\right)=\frac{d}{d t}\left[\Phi_{\epsilon}\left(T_{\epsilon S}\right)-\left(w_{2}-273\right) \gamma_{\epsilon}\left(T_{\epsilon S}\right)\right]+\gamma_{\epsilon}\left(T_{\epsilon S}\right) w_{2 t}
$$

for $\Phi_{\epsilon}: \mathbb{R} \rightarrow \mathbb{R}$ defined as follows

- $\quad$ If $u<273$

$$
\Phi_{\epsilon}(u)=\frac{1}{2} \gamma_{1}(u-273)^{2},
$$


- $\quad$ if $273 \leq u \leq 273+\epsilon$,

$$
\Phi_{\epsilon}(u)=-\left(\left(\gamma_{2}-\gamma_{1}\right) \epsilon+2 L\right) \frac{3(u-273)^{4}}{4 \epsilon^{3}}+\left(3 L+2\left(\gamma_{2}-\gamma_{1}\right) \epsilon\right) \frac{2(u-273)^{3}}{3 \epsilon^{2}}+\frac{\gamma_{1}}{2}(u-273)^{2},
$$

- $\quad$ if $u>273+\epsilon$,

$$
\Phi_{\epsilon}(u)=\frac{\gamma_{2}}{2}(u-273)^{2}+\frac{1}{12}\left(\gamma_{2}-\gamma_{1}\right) \epsilon^{2}+\frac{1}{2} \epsilon L .
$$

$\Phi_{\epsilon}$ verifies that $\Phi_{\epsilon}^{\prime}(u)=\gamma_{\epsilon}^{\prime}(u)(u-273)$ for all $u \in \mathbb{R}$. Notice that

$$
\left|\gamma_{\epsilon}\left(T_{\epsilon S}\right) w_{2 t}\right| \leq c
$$

Therefore, thanks to Lemma 1 we have

$$
\frac{d}{d t} \int_{\mathcal{M}}\left[\Phi_{\epsilon}\left(T_{\epsilon s}\right)-\hat{w}_{2} \gamma_{\epsilon}\left(T_{\epsilon s}\right)\right] d A+\int_{\mathcal{M}} k_{2}(x)\left|\nabla\left(T_{\epsilon s}-w_{2}\right)\right|^{2} d A \leq c .
$$

where $\hat{w}_{2}=w_{2}-273$. After integration over $(0, t)$, and thanks to Lemma 1 we obtain,

$$
\int_{0}^{T} \int_{\mathcal{M}}\left(\left|\nabla T_{\epsilon s}\right|^{2}+T_{\epsilon s}^{2}\right) d A d t \leq c(T+1)
$$

and the proof ends.

Lemma 3. Let $\left(T_{\epsilon v}, \Gamma_{\epsilon}\right)$ the solution to (10), and $T_{\epsilon s}=\varphi_{\epsilon}\left(\Gamma_{\epsilon}\right)$ then, there exist constants $c_{0}$ and $c_{1}$ independent of $\epsilon$ and $T$ such that

$$
\int_{0}^{T} \int_{\mathcal{M}}\left(\left|T_{\epsilon v t}\right|^{2}+\left|T_{\epsilon s t}\right|^{2}\right) d A d t \leq c_{0} T+c_{1}
$$

and

$$
\int_{\mathcal{M}}\left(\left|\nabla T_{\epsilon v}\right|^{2}+\left|\nabla T_{\epsilon s}\right|^{2}\right) d A \leq c_{0} T+c_{1}
$$

Proof. We multiply the first two equations in (10) by $T_{\epsilon v t}-w_{1 t}$ and $T_{\epsilon s t}-w_{2 t}$ respectively to obtain

$$
C_{v} \int_{\mathcal{M}}\left|T_{\epsilon v t}\right|^{2} d A+\frac{1}{2} \frac{d}{d t} \int_{\mathcal{M}} k_{1}(x)\left|\nabla T_{\epsilon v}\right|^{2} d A \leq \int_{\mathcal{M}} F_{1} T_{\epsilon v t} d A+\int_{\mathcal{M}} k_{1}(x) \nabla T_{\epsilon v} \nabla w_{1 t} d A,
$$

and

$$
\int_{\mathcal{M}} \gamma_{\epsilon}^{\prime}\left|T_{\epsilon s t}\right|^{2} d A+\frac{1}{2} \frac{d}{d t} \int_{\mathcal{M}} k_{2}(x)\left|\nabla T_{\epsilon s}\right|^{2} d A \leq \int_{\mathcal{M}} F_{2} T_{\epsilon s t} d A+\int_{\mathcal{M}} k_{2}(x) \nabla T_{\epsilon s} \nabla w_{2 t} d A .
$$

Since

$$
\gamma_{\epsilon}^{\prime}>\min \left\{\gamma_{1}, \gamma_{2}\right\}
$$

and thanks to Young inequality we get

$$
\begin{gathered}
\frac{C_{v}}{2} \int_{\mathcal{M}}\left|T_{\epsilon v t}\right|^{2} d A+\frac{1}{2} \frac{d}{d t} \int_{\mathcal{M}} k_{1}(x)\left|\nabla T_{\epsilon v}\right|^{2} d A \leq c \int_{\mathcal{M}}\left|F_{1}\right|^{2} d A \\
+c \int_{\mathcal{M}}\left|\nabla T_{\epsilon v}\right|^{2} d A+c \int_{\mathcal{M}}\left|\nabla w_{1 t}\right|^{2} d A
\end{gathered}
$$

and

$$
\begin{gathered}
\min \left\{\gamma_{1}, \gamma_{2}\right\} \int_{\mathcal{M}}\left|T_{\epsilon s t}\right|^{2} d A+\frac{1}{2} \frac{d}{d t} \int_{\mathcal{M}} k_{2}(x)\left|\nabla T_{\epsilon s}\right|^{2} d A \leq c \int_{\mathcal{M}}\left|F_{2}\right|^{2} d A \\
+c \int_{\mathcal{M}}\left|\nabla T_{\epsilon s}\right|^{2} d A+c \int_{\mathcal{M}}\left|\nabla w_{2 t}\right|^{2} d A .
\end{gathered}
$$


After integration over $(0, T)$ and thanks to previous lemmas and assumption $\mathcal{H}_{g}$, we get, for $T<\infty$

$$
\int_{0}^{T} \int_{\mathcal{M}}\left|T_{\epsilon v t}\right|^{2} d A+\int_{\mathcal{M}}\left|\nabla T_{\epsilon v}\right|^{2} d A \leq c_{0} T+c_{1}
$$

and

$$
\int_{0}^{T} \int_{\mathcal{M}}\left|T_{\epsilon s t}\right|^{2} d A+\int_{\mathcal{M}}\left|\nabla T_{\epsilon s}\right|^{2} d A \leq c_{0} T+c_{1}
$$

which ends the proof.

Proof of Theorem 1. The proof is divided into two different steps. In the first step we prove the existence and uniqueness of solution to the approximated problem (10). In the second step we prove the convergence of the approximated solution to (10) to the solution of (5).

Step 1. We consider the equation satisfied by $\left(T_{\epsilon v}, \Gamma_{\epsilon}\right)$,

$$
\begin{aligned}
& C_{v} \frac{\partial T_{\epsilon v}}{\partial t}-\operatorname{div}\left(k_{1}(x) \nabla T_{\epsilon v}\right)=F_{1}\left(T_{\epsilon v}, \varphi_{\epsilon}\left(\Gamma_{\epsilon}\right)\right), \\
& \frac{\partial \Gamma_{\epsilon}}{\partial t}-\operatorname{div}\left(k_{2}(x) \nabla \varphi_{\epsilon}\left(\Gamma_{\epsilon}\right)\right)=F_{2}\left(T_{\epsilon v}, \varphi_{\epsilon}\left(\Gamma_{\epsilon}\right)\right),
\end{aligned}
$$

with boundary conditions

$$
T_{\epsilon v}=g_{v}, \quad \Gamma_{\epsilon}=\gamma_{\epsilon}\left(g_{s}\right), \quad \text { in } \partial \mathcal{M}
$$

and initial data

$$
T_{\epsilon v}=T_{v 0}, \quad \Gamma_{\epsilon}(0, x)=\gamma_{\epsilon}\left(T_{s 0}\right), \quad \text { in } \partial \mathcal{M} .
$$

We construct a fixed point argument in the following way. Let

$$
\mathcal{A}:=\left\{u \in L^{2}\left(0, T: L^{2}(\mathcal{M})\right), \quad|u| \leq c_{\infty}\right\}
$$

where $c_{\infty}$ is defined in Lemma 1. Let

$$
E_{\epsilon}: \mathcal{A} \rightarrow L^{2}\left(0, T: L^{2}(\mathcal{M})\right)
$$

be defined by

$$
E_{\epsilon}\left(\hat{T}_{\epsilon v}, \hat{\Gamma}_{\epsilon}\right)=\left(T_{\epsilon v}, \Gamma_{\epsilon}\right),
$$

where $\left(T_{\epsilon v}, \Gamma_{\epsilon}\right)$ is the solution of the $\epsilon$-problem

$$
\begin{gathered}
C_{v} \frac{\partial T_{\epsilon v}}{\partial t}-\operatorname{div}\left(k_{1}(x) \nabla T_{\epsilon v}\right)=F_{1}\left(\hat{T}_{\epsilon v}, \varphi_{\epsilon}\left(\hat{\Gamma}_{\epsilon}\right)\right), \\
\frac{\partial \Gamma_{\epsilon}}{\partial t}-\operatorname{div}\left(k_{2}(x) \varphi_{\epsilon}^{\prime}\left(\hat{\Gamma}_{\epsilon}\right) \nabla \Gamma_{\epsilon}\right)=F_{2}\left(\hat{T}_{\epsilon v}, \varphi_{\epsilon}\left(\hat{\Gamma}_{\epsilon}\right)\right),
\end{gathered}
$$

with boundary conditions (15) and initial data (16).

Notice that, since $\hat{F}_{1}$ and $\hat{F}_{2}$ are uniformly bounded,

$$
0<\frac{1}{c}<\varphi_{\epsilon}^{\prime}<c<\infty,
$$


$T_{0 v}, T_{0 s} \in H^{1}(\mathcal{M})$, and $g_{v}, g_{s} \in C^{2}((0, T) \times \partial \mathcal{M})$ we have that there exists a unique solution to (17), (18) satisfying

$$
\int_{0}^{T} \int_{\mathcal{M}}\left[\left|T_{\epsilon v t}\right|^{2}+\left|\Gamma_{\epsilon t}\right|^{2}\right] d A d t+\int_{\mathcal{M}}\left[\left|\nabla T_{\epsilon v}\right|^{2}+\left|\nabla \Gamma_{\epsilon}\right|^{2}\right] d A \leq c(\epsilon),
$$

and

$$
\left\|T_{\epsilon v}\right\|_{L^{\infty}(\mathcal{M})},\left\|\Gamma_{\epsilon}\right\|_{L^{\infty}(\mathcal{M})} \leq c_{\infty}
$$

for time $T$ small enough. The proof of the estimates (19) and (20) is similar to the proofs of Lemmas 2 and 3 therefore we omit the details.

Thanks to the Aubin-Lions-Simon Lemma (see Simon [19])

$$
\left(T_{\epsilon v}, \Gamma_{\epsilon}\right) \in C\left(0, T: L^{2}(\mathcal{M})\right)^{2}
$$

and (19) and (20) yields $E_{\epsilon}(\mathcal{A}) \subset \mathcal{A}$. Standard computations, shows the continuity of

$$
E_{\epsilon}: \mathcal{A} \rightarrow\left[L^{\infty}\left(0, T: H^{1}(\mathcal{M})\right) \cap H^{1}\left(0, T: L^{2}(\mathcal{M})\right) \cap L^{\infty}\left(\mathcal{M}_{T}\right)\right]^{2} .
$$

Since

$$
H^{1}(\mathcal{M}) \hookrightarrow L^{p}(\mathcal{M}), \quad p<\infty
$$

is a compact embedding, we have that

$$
L^{\infty}\left(0, T: H^{1}(\mathcal{M})\right) \cap H^{1}\left(0, T: L^{2}(\mathcal{M})\right) \cap L^{\infty}\left(\mathcal{M}_{T}\right) \hookrightarrow L^{p}\left(0, T: L^{p}(\mathcal{M})\right)
$$

for any $p<\infty$ is a compact embedding as well as to $C\left(0, T: L^{p}(\mathcal{M})\right)$. Therefore, for any sequence $\left(T_{j \epsilon v}, \Gamma_{j \epsilon}\right)(j=1 \ldots \infty)$ defined by

$$
\left(T_{j \epsilon v}, \Gamma_{j \epsilon}\right)=E_{\epsilon}\left(T_{(j-1) \epsilon v}, \Gamma_{(j-1) \epsilon}\right)
$$

for

$$
\left(T_{1 \epsilon v}, \Gamma_{1 \epsilon}\right) \in \mathcal{A},
$$

we have that there exists a sub-sequence $\left(T_{n \in v}, \gamma_{n \epsilon}\right)$ such that

$$
\left(T_{n \epsilon v}, \Gamma_{n \epsilon}\right) \rightarrow\left(T_{\epsilon v}^{*}, \Gamma_{\epsilon}^{*}\right)
$$

in $L^{p}\left(0, T: L^{p}(\mathcal{M})\right)$ strong and

$$
\left(T_{n \epsilon v}, \Gamma_{n \epsilon}\right) \rightarrow\left(T_{\epsilon v}^{*}, \Gamma_{\epsilon}^{*}\right)
$$

weakly in $L^{p}\left(0, T: H^{1}(\mathcal{M})\right)$. Then, it results that

$$
\int_{0}^{T} \int_{\mathcal{M}} k_{2}(x) \varphi_{\epsilon}^{\prime}\left(\Gamma_{(n-1) \epsilon}\right) \nabla \Gamma_{n \epsilon} \nabla \psi_{2} d A d t \rightarrow \int_{0}^{T} \int_{\mathcal{M}} k_{2}(x) \varphi_{\epsilon}^{\prime}\left(\Gamma_{\epsilon}^{*}\right) \nabla \Gamma_{\epsilon}^{*} \nabla \psi_{2} d A d t
$$

and

$$
\int_{0}^{T} \int_{\mathcal{M}}\left|\varphi_{\epsilon}\left(\Gamma_{(n-1) \epsilon}\right)\right|^{q} \varphi_{\epsilon}\left(\Gamma_{(n-1) \epsilon}\right) \psi_{2} d A d t \rightarrow \int_{0}^{T} \int_{\mathcal{M}}\left|\varphi_{\epsilon}\left(\Gamma_{\epsilon}^{*}\right)\right|^{q} \varphi_{\epsilon}\left(\Gamma_{\epsilon}^{*}\right) \psi_{2} d A d t
$$

Therefore $\Gamma_{\epsilon}^{*}$ satisfies the weak formulation of (10) given in (2) in $(0, T)$. In view of Lemma 1 we may extend the solution up to $T<\infty$.

Uniqueness of solutions of (10) is a consequence of regularity of the functions $\gamma_{\epsilon}, F_{1}$ and $F_{2}$. 
Step 2. Let $\left(T_{\epsilon v}, \Gamma_{\epsilon}\right)$ be the solution to (10), then, we denote by $T_{\epsilon S}$ the inverse of $\Gamma_{\epsilon}^{*}$, i.e.,

$$
T_{\epsilon s}=\varphi_{\epsilon}\left(\Gamma_{\epsilon}^{*}\right)
$$

Thanks to Lemmas 1-3 and Aubin-Lions-Simon Lemma, there exists a subsequence, $\left(\tilde{T}_{\epsilon v}, \tilde{T}_{\epsilon s}\right)$ such that

$$
\tilde{T}_{\epsilon s}=\varphi_{\epsilon}\left(\tilde{\Gamma}_{\epsilon}^{*}\right)
$$

satisfying

$$
\left(\tilde{T}_{\epsilon v}, \tilde{T}_{\epsilon s}\right) \rightarrow\left(T_{v}^{*}, T_{s}^{*}\right)
$$

strongly in $L^{p}\left(0, T: L^{p}(\mathcal{M})\right)$ and $C\left(0, T: L^{p}(\mathcal{M})\right)$ for any $p<\infty$ and

$$
\left(\tilde{T}_{\epsilon v}, \tilde{T}_{\epsilon s}\right) \rightarrow\left(T_{v}^{*}, T_{s}^{*}\right)
$$

weakly in $L^{p}\left(0, T: H^{1}(\mathcal{M})\right)$ for any $p<\infty$.

Since any $Z_{1} \in \gamma\left(\tilde{T}_{\epsilon s}\right)$ and $Z_{2} \in \gamma\left(\tilde{T}_{\epsilon S}-\epsilon\right)$ are uniformly bounded in $L^{\infty}\left(\mathcal{M}_{T}\right)$, there exist $\bar{\gamma}_{\epsilon}$ and $\underline{\gamma}_{\epsilon}$ such that

$$
\begin{gathered}
\bar{\gamma}_{\epsilon} \in \gamma\left(\tilde{T}_{\epsilon S}\right) \\
\underline{\gamma}_{\epsilon} \in \gamma\left(\tilde{T}_{\epsilon S}-\epsilon\right)
\end{gathered}
$$

satisfying

$$
\underline{\gamma}_{\epsilon} \leq \gamma_{\epsilon}\left(\tilde{T}_{\epsilon S}\right) \leq \bar{\gamma}_{\epsilon}
$$

In view of the boundedness in $L^{\infty}\left(\mathcal{M}_{T}\right)$ of $\bar{\gamma}_{\epsilon}$ and $\underline{\gamma}_{\epsilon}$ we have that there exists a subsequence of $\epsilon$ (denoted with the same sub index) such that

$$
\bar{\gamma}_{\epsilon} \rightarrow \bar{\gamma}^{*}
$$

and

$$
\underline{\gamma}_{\epsilon} \rightarrow \underline{\gamma}^{*}
$$

weakly in $L^{p}\left(\mathcal{M}_{T}\right)$ for any $p<\infty$.

Thanks to [20] Proposition 20.32, p. 300 (see also [16]), since $\gamma$ is maximal monotone, we have

$$
\underline{\gamma}^{*}, \bar{\gamma}^{*} \in \gamma\left(T_{s}^{*}\right) \text {. }
$$

In view of the boundedness of $\gamma_{\epsilon}\left(\tilde{T}_{\epsilon S}\right)$ there exists a subsequence, denoted by $\tilde{\gamma}_{\epsilon}$ such that

$$
\tilde{\gamma}_{\epsilon} \rightarrow \gamma^{* *}
$$

weakly in $L^{p}\left(\mathcal{M}_{T}\right)$ for any $p<\infty$. The inequality (21) proves

$$
\underline{\gamma}^{*} \leq \gamma^{* *} \leq \bar{\gamma}^{*}
$$

Since $\gamma$ is maximal, (24) and (25) prove

$$
\gamma^{* *} \in \gamma\left(T_{s}^{*}\right) .
$$

Since $\tilde{T}_{\epsilon v}$ converges strongly in $C\left(0, T: L^{2}(\mathcal{M})\right)$ and $\gamma$ is maximal monotone we proceed as before to obtain the convergence of a subsequence (of the previous subsequence) of $\gamma_{\epsilon}\left(\tilde{T}_{\epsilon s}(T, \cdot)\right)$ to $\gamma^{* * *} \in \gamma\left(T_{s}^{*}(T, \cdot)\right)$.

We now take limits in the weak formulation of problem (10) to conclude the result. 


\section{Discussion}

A two-layers model has been proposed to analyze the thermal behaviour of green roofs. The model is based on an energy balance on the vegetation layer and the soil layer. The model incorporates the evapotranspiration, that is, the combination of soil evaporation and plants transpiration, this effect is one of the differences between a green roof and a conventional roof. The modeling has considered some simplifications in the reaction terms $F_{1}$ and $F_{2}$, the case where the reaction terms are more general functions will be considered in the future including non-polynomial growth terms. The shape of the green roof is defined in a two dimensional spatial domain which leads to formulate the model as a system of partial differential equations on manifolds. One of the difficulties of the mathematical analysis of the model is the multivalued term $\gamma\left(T_{S}\right)$ in the balance on the substratum. The multivalued term and the coefficient $C_{v}$ present a dependence of the humidity $w$, assumed known. Further development must consider $w$ as unknown of a third equation, which will allow us to decrease the data collection.

We have proved the existence of solutions (temperature in every layer) in a suitable functional space and we have obtained some estimates of them. This mathematical treatment open new questions about this mathematical model. The model will allow to analyze the impact of fluctuations of the parameters, among them, the leaf area index $(\mathrm{LAI})$, fractional vegetation coverage $\left(\sigma_{v}\right)$.

\section{Conclusions}

In this work, we have proposed and analyzed a mathematical model of the thermal behavior of the green roof. The system consists of two coupled parabolic equations describing the temperature in a vegetation layer and the temperature in the substrate. Two features of this nonlinear model are remarkable in comparison with other previous models in the study of green roofs: the space domain which is a surface (and so, a two dimensional space variable) and the term $\gamma$ to describe the effect of the change of phases in the substratum. The other terms in the energy balance model were expressed as polynomials with power less or equal to four.

The second result of the article is the mathematical proof of the existence of solutions which is presented in detail. Due to the shape of $\gamma$, classical solutions are not expected if the temperature falls bellow $273 \mathrm{~K}$, therefore weak solutions have to be introduced.

Future work of this two-layer energy balance model will be the analysis of the relevant parameters (e.g., LAI), the inclusion of non-polynomial reaction terms and the numerical approximation of the solutions.

Author Contributions: Conceptualization, J.I.T., L.T. and M.L.V.; methodology, J.I.T., L.T. and M.L.V.; formal analysis, J.I.T., L.T. and M.L.V.; investigation, J.I.T., L.T. and M.L.V.; resources, J.I.T., L.T. and M.L.V.; writing-original draft preparation, J.I.T., L.T. and M.L.V.; writing-review and editing, J.I.T., L.T. and M.L.V.; visualization, J.I.T., L.T. and M.L.V.; supervision, J.I.T., L.T. and M.L.V.; project administration, J.I.T., L.T. and M.L.V.; funding acquisition, J.I.T., L.T. All authors have read and agreed to the published version of the manuscript.

Funding: The research of the first two authors is partially supported by the project MTM2017-83391-P Ministerio de Ciencia e Innovación, Spain. Second author is also supported by project MTM2017-85449-P, Ministerio de Ciencia e Innovación, Spain.

Conflicts of Interest: The authors declare no conflict of interest.

\section{References}

1. Kumar, R.; Kaushik, S.C. Performance evaluation of green roof and shading for thermal protection of buildings. Build. Environ. 2005, 40, 1505-1511. [CrossRef]

2. Jaffal, I.; Belarbi, R.; Ouldboukhitine, S.E. A comprehensive study to the impact of green roofs on building energy performance. Renew. Energy 2012, 43, 157-164. [CrossRef]

3. Zhang, G.; He, B.J.; Dewancker, B.J. The maintenance of prefabricated green roofs for preserving cooling performance: A field measurement in the subtropical city of Hangzhou, China. Sustain. Cities Soc. 2020, 102314. [CrossRef] 
4. Quezada-García, S.; Espinosa-Paredes, G.; Polo-Labarrios, M.A.; Espinosa-Martínez, E.G.; Escobedo-Izquierdo, M.A. Green roof heat and mass transfer mathematical models: A review. Build. Environ. 2020, 170, 106634. [CrossRef]

5. Palomo del Barrio, E. Analysis of the green roofs cooling potential in buildings. Energy Build. 1998, 27, $179-193$. [CrossRef]

6. Sailor, D.J. A green roof model for building energy simulation programs. Energy Build. 2008, 40, 1466-1478. [CrossRef]

7. Gholami, M.; Barbaresi, A.; Tassinari, P.; Bovo, M.; Torreggiani, D. A Comparison of Energy and Thermal Performance of Rooftop Greenhouses and Green Roofs in Mediterranean Climate: A Hygrothermal Assessment in WUFI. Energies 2020, 13, 2030. [CrossRef]

8. Sellers, P.J.; Mintz, Y.; Sud, Y.C.; Dalcher, A. A simple Biosphere model for use within General Circulation Model. J. Atmos. Sci. 1986, 43, 505-531. [CrossRef]

9. De Vries, D.A. Thermal properties of soils. In Physics of Plant Environment; Van Wijk, W.R., Ed.; John Wiley Sons: New York, NY, USA, 1963; pp. 210-235.

10. Frankenstein, S.; Koenig, G. FASST Vegetation Models. ERDC/CRREL TR-04-25 2004. Available online: https:/ / www.researchgate.net/publication/235157815_Fast_All-Season_Soil_STrength_FASST (accessed on 17 September 2020).

11. Henderson-Sellers, B. A new formula for latent heat of vaporization of water as a function of temperature. Q. J. R. Metal. Soc. 1984, 110, 1186-1190. [CrossRef]

12. Díaz, J.I.; Hidalgo, A.; Tello, L. Multiple solutions and numerical analysis to the dynamic and stationary models coupling a delayed energy balance model involving latent heat and discontinuous albedo with a deep ocean. Proc. R. Soc. Lond. A Math. Phys. Eng. Sci. 2014, 470, 20140376.

13. Hidalgo, A.; Tello, L. On a climatological energy balance model with continents distribution. Discret. Contin. Dyn. Syst. 2015, 35, 1503-1519. [CrossRef]

14. Aubin, T. Nonlinear Analysis on Manifolds. Monge-Ampere Equations; Springer: New York, NY, USA, 1982.

15. Díaz, J.I.; Tello, L. A nonlinear parabolic problem on a Riemannian manifold without boundary arising in Climatology. Collect. Math. 1999, 50, 19-51.

16. Brezis, H. Operateurs Maximaux Monotones et Semigroupes de Contractions dans les Espaces de Hilbert; Elsevier: Amsterdam, The Netherlands, 1973.

17. Hetzer, G.; Tello, L. On a reaction diffusion system arising in climatology. Dyn. Syst. Appl. 2002, 11, 381-402.

18. Gilbarg, D.; Trudinger, N. Elliptic Partial Differential Equations of Second Order; Springer-Verlag: Berlin/Heidelberg, Germany; New York, NY, USA, 1977.

19. Simon, J. Compact sets in $L^{p}(0, T ; B)$. Ann. Mat. Pura Appl. Sér. IV 1987, CXLVI, 65-96.

20. Bauschke, H.H.; Combettes, P.L. Convex Analysis and Monotone Operator Theory in Hilbert Spaces; Springer: New York, NY, USA, 2011.

(C) 2020 by the authors. Licensee MDPI, Basel, Switzerland. This article is an open access article distributed under the terms and conditions of the Creative Commons Attribution (CC BY) license (http:/ / creativecommons.org/licenses/by/4.0/). 